\title{
Programa Nacional de Alimentação Escolar: estratégias para enfrentar a insegurança alimentar durante e após a COVID-19
}

\author{
Ana Laura Benevenuto de Amorim 1 \\ José Raimundo Sousa Ribeiro Junior ${ }^{2}$ \\ Daniel Henrique Bandoni ${ }^{3}$ \\ ${ }^{1}$ Universidade Federal de São Paulo / Programa Interdisciplinar em Ciências da Saúde, Santos / SP - Brasil \\ ${ }^{2}$ Universidade Federal de São Paulo / Centro de Práticas e Pesquisa em Nutrição e Alimentação Coletiva, Santos / SP - Brasil \\ ${ }^{3}$ Universidade Federal de São Paulo / Departamento de Saúde, Clínica e Instituições, Instituto de Saúde e Sociedade, Santos / \\ SP - Brasil
}

Este artigo tem como objetivo analisar a contribuição do Programa Nacional de Alimentação Escolar (PNAE) para o enfrentamento da fome e da insegurança alimentar (IA), ao propor um conjunto de estratégias para que o PNAE possa garantir a alimentação dos escolares neste momento de crise. Realizamos um diagnóstico da IA e da fome no Brasil, analisamos o processo de enfraquecimento das políticas de segurança alimentar e nutricional, consideramos a evolução histórica do PNAE e sua situação atual e, com base nessas reflexões, apresentamos um conjunto de propostas, avaliando seus impactos no orçamento destinado ao PNAE. Apresentamos as seguintes estratégias para o período em que as aulas estiverem suspensas: distribuição de kits ou refeições para escolares, se possível mantendo o caráter universal da política ou beneficiando estudantes das famílias elegíveis para receber o Auxílio Emergencial; ampliar o valor repassado pelo PNAE para os municípios com Índice de Desenvolvimento Humano (IDH) baixo e muito baixo; manter e incentivar a aquisição de alimentos da agricultura familiar. Após o retorno das aulas, sugerimos as seguintes estratégias: levantamento da IA entre os estudantes, manutenção da equidade da política por meio da ampliação do valor do repasse para municípios com IDH baixo e muito baixo, além de atendimento dos escolares de famílias em IA durante as férias e o recesso escolar.

Palavras-chave: COVID-19; Programa Nacional de Alimentação Escolar (PNAE); alimentação escolar; segurança alimentar e nutricional.

\section{Programa Nacional de Alimentación Escolar: estrategias para enfrentar la inseguridad alimentaria durante y después de la COVID-19}

Este artículo tiene como objetivo analizar la contribución del Programa Nacional de Alimentación Escolar (PNAE) en la lucha contra el hambre y la inseguridad alimentaria (IA) y proponer un conjunto de estrategias para que el PNAE pueda garantizar la alimentación de los estudiantes en este momento de crisis. Para tanto, realizamos un diagnóstico de la IA y el hambre en Brasil, analizamos el debilitamiento de las políticas de seguridad alimentaria y nutricional, consideramos la evolución histórica del PNAE y su situación actual. Con base en estas reflexiones, presentamos un conjunto de propuestas evaluando sus impactos en el presupuesto asignado al PNAE. Presentamos las siguientes estrategias para el período en que las clases estén suspendidas: distribución de kits de alimentos o comidas a los alumnos, manteniendo, si fuera posible, el carácter universal de la política o beneficiando a los estudiantes de familias elegibles para recibir la Ayuda de Emergencia debido a la COVID-19; aumentar el monto transferido por el PNAE a los municipios con un índice de desarrollo humano (IDH) bajo y muy bajo y; mantener e incentivar la compra de alimentos a la agricultura familiar. Después del regreso de las clases, sugerimos las siguientes estrategias: encuesta de IA entre los estudiantes; mantenimiento de la equidad de la política mediante el aumento del monto de la transferencia a municipios con IDH bajo y muy bajo; y asistencia a los estudiantes de familias en situación de IA durante las vacaciones y el receso escolar.

Palabras clave: COVID-19; Programa Nacional de Alimentação Escolar (PNAE); alimentación escolar; seguridad alimentaria y nutricional. 


\section{National school feeding program: strategies to overcome food insecurities during and after the COVID-19 pandemic}

This article aims to analyze the contribution of the National School Feeding Program (PNAE), in the fight against hunger and food insecurity (FI) and to propose a set of strategies to guarantee that students will continue to receive food during the COVID-19 pandemic. Diagnosis of FI and hunger in Brazil was carried out, analyzing the weakening of Food and Nutritional Security Policies, considering the historical evolution of PNAE and its current situation, and from this analysis, a set of proposals were drawn up, assessing their impacts on the PNAE's budget. We present the following strategies for the period of school closures: distribution of meals for students, maintaining, if possible, the universal character of the policy or benefiting students from families eligible to receive Emergency Aid due to COVID-19; expand the amount transferred by the PNAE to municipalities with a low and very low Human Development Index (HDI) and; maintenance and incentive to purchase food from family farming. When schools re-open, we suggest the following strategies: FI survey among students; maintenance of the equity of the policy by expanding the transfer amount to municipalities with low and very low HDI, and; attendance of students from families in FI during the vacations.

Keywords: COVID-19; Programa Nacional de Alimentação Escolar (PNAE); school feeding; food and nutrition security.

\section{INTRODUÇÃO}

Uma das principais características das políticas voltadas à garantia da segurança alimentar implementadas no Brasil foi a descontinuidade. Poucas delas deixaram de ser políticas associadas a governos específicos e se tornaram políticas de Estado amparadas pela Constituição, como é o caso do Programa Nacional de Alimentação Escolar (PNAE) (Vasconcelos, 2005).

Denominado inicialmente de Campanha Nacional da Merenda Escolar, em seus primeiros anos o PNAE era assistencial e visava reduzir a desnutrição mediante oferta de suplementação alimentar com foco nas áreas mais pobres do país (Peixinho, 2013). A Constituição Federal de 1988, ao universalizar o direito à alimentação escolar ${ }^{1}$ e definir que este deve ser garantido pelos três níveis de governo, foi o impulso decisivo para a transformação do PNAE em política de Estado. Desde então, outras leis foram responsáveis pelo crescimento e pelo fortalecimento do programa, que hoje atende aproximadamente 40 milhões de escolares em todo o país e tem como objetivo contribuir para o crescimento e o desenvolvimento biopsicossocial, a aprendizagem, o rendimento escolar e a formação de hábitos alimentares saudáveis dos alunos, por meio de ações de educação alimentar e nutricional e da oferta de refeições que atendam às necessidades nutricionais durante o período letivo (Lei n $11.947,2009$ ).

Estudos apontam a influência de aspectos socioeconômicos na adesão à alimentação escolar, evidenciando sua importância sobretudo para determinados grupos de escolares. Autores de diferentes pesquisas identificaram que o consumo da alimentação escolar é maior para meninos, residentes nas regiões Norte, Nordeste e Centro-Oeste, na área rural e entre pretos e pardos; e menor no estado de São Paulo, nas áreas metropolitanas, e que tende a diminuir com a idade e o aumento da renda domiciliar per capita (Hoffmann, 2012). Outro achado é que escolares do sexo masculino e pardos, que não moram na capital e cujas mães têm baixa escolaridade, apresentam mais chances de consumir regularmente a alimentação escolar (Locatelli, Canela, \& Bandoni, 2017).

\footnotetext{
${ }^{1}$ Com a Constituição Federal, os alunos do ensino fundamental passaram a ter o direito à alimentação escolar. Somente em 2009, com a Lei no 11.947, é que a alimentação escolar passou a atender todos os alunos matriculados na educação básica.
} 
Neste momento, por conta do isolamento social para o enfrentamento da pandemia da COVID-19, o PNAE se encontra frente a um enorme desafio para continuar com o fornecimento da alimentação escolar. Em muitos casos, a suspensão das aulas nas escolas públicas significou a interrupção ou a precarização do acesso à alimentação, o que não apenas impacta no orçamento das famílias, mas também prejudica quantitativa e qualitativamente a alimentação dos alunos.

Assim, entendemos que o PNAE tem um papel importantíssimo no combate à insegurança alimentar (IA) e a fome durante a pandemia, de modo que propomos um conjunto de estratégias a serem adotadas dentro do escopo desse programa. Para fundamentar nossas propostas, iniciamos a exposição apresentando um diagnóstico da IA e da fome no Brasil. Em seguida, analisamos o processo de enfraquecimento das políticas de segurança alimentar e nutricional. Na sequência, consideramos a evolução histórica do PNAE e sua situação atual. Por fim, propomos um conjunto de estratégias para que o PNAE possa garantir a alimentação dos estudantes durante e após o período de isolamento social.

\section{A INSEGURANÇA ALIMENTAR E A FOME NO BRASIL}

Um dos primeiros desafios que se impõem para enfrentar o problema da fome e da insegurança alimentar é a própria quantificação do fenômeno. Diferentes definições e metodologias produzem dados distintos e, por vezes, antagônicos. Desde os anos 1970, a Organização das Nações Unidas para a Alimentação e a Agricultura (FAO) utiliza o indicador de prevalência de desnutrição para estimar a quantidade de pessoas famintas no mundo. Apesar de ser uma medida importante para identificar os casos mais agudos de fome, esse indicador se mostrou inadequado para captar a fome em toda a sua extensão.

Desse modo, a partir dos anos 1980, diferentes pesquisadores buscaram desenvolver uma nova metodologia de quantificação da fome (Radimer, Olson, Green, Campbell, \& Habicht, 1992; Wehler, Scoot, \& Anderson, 1992), que levou ao desenvolvimento da Escala de Segurança Alimentar Domiciliar, adotada pelo Serviço de Alimentação e Nutrição do Departamento de Agricultura dos Estados Unidos a partir de 1995 (Bickel et al., 2000). Em 2003, foi iniciada a tradução e a adaptação da Escala de Segurança Alimentar e Domiciliar estadunidense, com o objetivo de contribuir para o recém-lançado Programa Fome Zero, por meio da elaboração de “indicadores diretos de quantificação da população sujeita a diferentes graus de insegurança alimentar, apropriados, também, para o acompanhamento e avaliação de suas ações e estratégias" (Segall-Correa \& Marin-Leon, 2009).

Uma vez validada, a Escala Brasileira de Insegurança Alimentar (Ebia) foi utilizada pelo Instituto Brasileiro de Geografia e Estatística (IBGE) no suplemento de Segurança Alimentar da Pesquisa Nacional por Amostra de Domicílio (PNAD) em 2004, 2009 e 2013, fornecendo os dados mais confiáveis que temos sobre o acesso aos alimentos no Brasil. A produção desses dados permitiu uma melhor compreensão do perfil da insegurança alimentar no país, tanto no que se refere à sua magnitude e severidade quanto com relação às suas principais determinantes e distribuição espacial.

Entre 2004 e 2013, a quantidade de moradores em domicílios em situação de insegurança alimentar passou de pouco mais de 73 milhões (39,8\% da população) para aproximadamente 52 milhões $(25,8 \%)$. Se tomarmos os dados relativos aos domicílios em situação de IA moderada e grave, nos quais foi constatada restrição quantitativa de alimentos, a redução na quantidade de pessoas convivendo com a fome foi de cerca de 35,8 milhões (19,5\%) para 17,5 milhões de pessoas ([8,7\%] IBGE, 2014). 
Tais dados explicitam que, nesse período, houve melhora na situação alimentar dos brasileiros, mas também revelam que uma parcela significativa da população ainda encontrava acentuada dificuldade para se alimentar adequadamente. Dados de 2013 indicavam que, em 80\% dos domicílios em situação de IA, a renda mensal domiciliar per capita era de até 1 salário mínimo. Além disso, 34,2\% daqueles que tinham até 4 anos e 33,2\% daqueles que tinham entre 5 e 17 anos estavam em IA, o que reforça a importância do PNAE (IBGE, 2014).

\section{O ENFRAQUECIMENTO DAS POLÍTICAS DE SEGURANÇA ALIMENTAR E NUTRICIONAL}

Se a situação alimentar da população brasileira em 2013 demandava atenção do poder público, essa situação se agravou a partir de 2015. Desde então, houve crescimento da desocupação e da subutilização da força de trabalho, que atingiram, respectivamente, 6,9\% e 24,6\% da população em 2018 (IBGE, 2019). Em consonância com esse processo, entre 2014 e 2018, a proporção de pessoas vivendo abaixo da linha de pobreza - isto é, que vivem com menos 5,50 dólares por dia - passou de 22,8\% para 25,3\% (52,5 milhões), e a proporção de pessoas vivendo abaixo da linha de extrema pobreza - aquelas que vivem com até 1,90 dólar por dia - passou de 4,5\% para 6,5\% ([13,5 milhões] IBGE, 2019). Esse cenário demanda a ampliação e o fortalecimento das políticas de segurança alimentar e nutricional (SAN) para a garantia do Direito Humano à Alimentação Adequada ([DHAA] Lei no 11.346, 2006).

$\mathrm{O}$ que se observou em anos recentes, entretanto, foi o enfraquecimento dessas políticas pelo governo federal. Os processos abaixo ilustram esse enfraquecimento:

1. Desarticulação do Sistema Nacional de Segurança Alimentar e Nutricional (Sisan), por meio do gradativo abandono do Conselho Nacional de Segurança Alimentar e Nutricional (Consea) e da Câmara Interministerial de Segurança Alimentar e Nutricional (Caisan). Peças essenciais para o funcionamento do Sisan, ambos foram formalmente extintos por uma medida provisória do presidente Jair Bolsonaro.

2. Não realização da Conferência Nacional de Segurança Alimentar e Nutricional, instância responsável pela indicação de diretrizes e prioridades da Política e do Plano Nacional de Segurança Alimentar (Plansan). Com efeito, o governo federal não elaborou o plano previsto para o período de 20202023.

3. O Programa Bolsa Família (PBF), principal programa de transferência de renda do país, teve orçamento e número de beneficiários reduzidos. Entre dezembro de 2018 e dezembro de 2019, houve diminuição de 1 milhão de famílias beneficiadas (de 14,1 para 13,1 milhões), e o orçamento original para 2020 apontava para uma continuidade nesse processo, com diminuição de $\mathrm{R} \$ 32,5$ para $\mathrm{R} \$ 29,5$ bilhões. $^{2}$

Dentro desse processo de enfraquecimento das políticas de SAN, destacam-se ainda os cortes orçamentários em outros programas importantes, como o Programa de Aquisição de Alimentos da Agricultura Familiar (PAA), e a diminuição dos estoques estratégicos de alimentos da Conab, essenciais para garantir uma Política de Garantia de Preços Mínimos, que protege os produtores rurais. Além

${ }^{2}$ Recuperado de https://www2.camara.leg.br/orcamento-da-uniao/raio-x-do-orcamento-previdencia/raio-x-do-orcamento-ploa-2020 
disso, esses estoques são úteis em situações de crise, pois podem ser utilizados para abastecer escolas e compor doações para a população que necessita de assistência alimentar.

\section{A EVOLUÇÃO DO PROGRAMA NACIONAL DE ALIMENTAÇÃO ESCOLAR}

Desde a Constituição Federal de 1988, a evolução do PNAE foi marcada por avanços que merecem destaque. O primeiro deles remete à descentralização de sua gestão, por meio da Lei no 8.913 , de 1994. Desse momento em diante, o Ministério da Educação (MEC) firmou convênios com estados e municípios - entidades executoras (EEx) - para o repasse de recursos financeiros, permitindo a regularização no fornecimento e a melhoria da qualidade das refeições. Atualmente, o PNAE é gerido pelo Fundo Nacional de Desenvolvimento da Educação (FNDE), autarquia vinculada ao MEC, que repassa para as EEx um valor fixo determinado pelo número de estudantes por modalidade de ensino, de acordo com o período de permanência na escola, considerando 200 dias letivos.

Em 2000, o PNAE instituiu a participação social por meio dos Conselhos de Alimentação Escolar (CAE), obrigatórios em todas as EEx e que tem papel de acompanhar e fiscalizar a execução do programa (Medida Provisória no $1.979-19,2000$ ).

Outros avanços significativos ocorreram com a revisão do marco legal do PNAE (Lei n 11.947, de 2009), que universalizou o Programa ao prever o fornecimento de alimentação escolar saudável e adequada para todos os alunos matriculados na educação básica. Além disso, estabeleceu que no mínimo 30\% dos recursos financeiros devem ser usados para a aquisição de gêneros alimentícios diretamente da agricultura familiar ( $\mathrm{AF}$ ), priorizando os assentamentos da reforma agrária, as comunidades tradicionais indígenas e quilombolas, bem como os alimentos orgânicos e/ou agroecológicos. Essa disposição tem como objetivo promover o desenvolvimento econômico, combater a desigualdade, minimizar o êxodo rural e garantir a SAN no campo (Assis, Priore, \& Franceschin, 2017). ${ }^{3}$

Por fim, nas últimas duas décadas, verificou-se uma ampliação dos recursos federais para o PNAE, em conformidade com o aumento do número de estudantes atendidos. No entanto, persiste o desafio de garantir uma alimentação saudável e variada com o valor que varia de $R \$ 0,32$ a $R \$ 2,00$ per capita por dia (Resolução $n^{\circ}$ 01, 2017). O PNAE prevê que esse valor seja suplementado pelas esferas estaduais e municipais, mas as desigualdades econômicas, sociais e regionais do país tornam a execução da política desigual, ainda que universal. Entidades Executoras com menos recursos tendem a despender valores menores com essa suplementação, o que muitas vezes inviabiliza o fornecimento de uma alimentação como prevê a lei.

\section{O PNAE FRENTE À PANDEMIA DO CORONAVÍRUS}

Como estratégia de enfrentamento a COVID-19 no Brasil, a partir do dia 23 de março de 2020, as redes municipais, estaduais e federais de ensino suspenderam as aulas e as atividades presenciais nas escolas de todo o país. Apesar de ser uma medida previsível, visto que outros países já enfrentavam

\footnotetext{
${ }^{3}$ Apesar do avanço nas compras de gêneros alimentícios da AF, dados disponibilizados pelo FNDE indicam que municípios ainda não conseguiram atingir os 30\% previstos em lei. O adquirido com AF em 2017 correspondeu a somente 17,46\% (FNDE, 2020).
} 
situações parecidas, quando ela ocorreu, não havia, por parte do FNDE, uma orientação sobre como as EEx deveriam proceder no fornecimento da alimentação escolar.

Dada a urgência da situação, os gestores adotaram medidas distintas para continuar fornecendo alimentos aos escolares e, sem respaldo legal, se viram impossibilitados de utilizar os recursos do PNAE para essas ações.

O respaldo legal veio somente em 7 de abril, por meio da Lei $\mathrm{n}^{\circ}$ 13.987, que autorizou, "em caráter excepcional, durante o período de suspensão das aulas em razão de situação de emergência ou calamidade pública, a distribuição de gêneros alimentícios adquiridos com recursos do PNAE aos pais ou responsáveis dos estudantes das escolas públicas da educação básica”. Dois dias depois, o FNDE publicou uma resolução que dispõe sobre a execução do PNAE durante a calamidade pública, acompanhada de uma cartilha que teve como objetivo orientar os gestores a lidar com o momento de crise (Resolução $n^{\circ}$ 02, 2020; Mapa \& MEC, 2020).

A partir desse momento, duas preocupações emergiram com relação ao funcionamento do PNAE durante e após o período de calamidade pública. A primeira delas se refere ao caráter universal do programa, pois o primeiro artigo da Resolução 02 dá autonomia às EEx para definir os critérios de elegibilidade para o recebimento de alimentos ou refeições adquiridos com recursos do PNAE (Resolução $n^{\circ}$ 02, 2020). Entende-se que tal posicionamento foi necessário, dada a dificuldade de preparação de kits com o recurso transferido exclusivamente pelo Governo Federal a todos os estudantes. Porém, em alguns casos, os critérios estabelecidos têm sido restritivos, privando muitos alunos que também precisam desses alimentos. É o caso, por exemplo, daquelas EEx que restringiram esse auxílio alimentar apenas às famílias que fazem parte do Programa Bolsa Família.

A segunda preocupação está relacionada à manutenção da aquisição dos alimentos da $\mathrm{AF}$ e à qualidade da alimentação fornecida. Apesar das dificuldades que a crise impõe, o PNAE deve continuar privilegiando a oferta de alimentação saudável e variada, com restrições e proibições de certos grupos de alimento. A compra de alimentos da AF, essencial para a promoção da segurança alimentar de produtores, consumidores (alunos), e que vem se consolidando há mais de 10 anos, cumpre um papel central para isso e deve ser valorizada.

\section{ESTRATÉGIAS PARA ENFRENTAR A INSEGURANÇA ALIMENTAR E A FOME DURANTE (E APÓS) A PANDEMIA DO CORONAVÍRUS}

O PNAE cumpre um importante papel para a garantia do DHAA dos estudantes matriculados na rede pública de ensino de todo o país, que representam $80 \%$ de toda a educação básica. Essa rede tem enorme capilaridade, e o programa já conta com a infraestrutura necessária para viabilizar as estratégias propostas, o que, por sua vez, permite que os recursos investidos cheguem rapidamente a quem necessita.

É importante ressaltar ainda que a rede pública de ensino está presente em todo o território nacional e se caracteriza por diferenças e desigualdades significativas. Assim, não é recomendável que sejam utilizadas estratégias idênticas para todos as EEx. O FNDE deve garantir que os gestores locais, em contato com o CAE, tenham respaldo legal e recursos financeiros para definir as estratégias mais adequadas para cada contexto.

Frente ao complexo cenário, dividimos o conjunto de estratégias em dois grupos. 


\subsection{Estratégias para o período em que as aulas estiverem suspensas}

\subsubsection{Fornecimento de kits de alimentos e/ou refeições}

A Resolução 02 prevê que os recursos do PNAE podem ser utilizados para oferecer kits de alimentos e/ou refeições (para viagem) durante o período de isolamento social. Para ambas as ações, estratégias que minimizem aglomerações e exposições de trabalhadores devem ser tomadas (Mapa \& MEC, 2020).

Refeições tendem a ter um custo unitário mais baixo e podem ser oferecidas diariamente para os estudantes, mas demandam a mobilização diária daqueles que preparam os alimentos e de pelo menos um membro da família dos estudantes. Já os kits de alimentos podem ser oferecidos semanal ou mensalmente, o que diminui a quantidade de deslocamentos necessários. No entanto, seu custo tende a ser mais alto, tornando-se um fator restritivo tanto para a qualidade e a quantidade dos alimentos ofertados quanto para a quantidade de estudantes que podem ser atendidos.

A escolha por uma dessas modalidades deve envolver um conjunto de critérios baseados na realidade local, dado que cada uma delas apresenta vantagens e desvantagens distintas.

Propomos a manutenção da universalidade do programa, mas entendemos que, na atual situação, alguns gestores terão de priorizar o atendimento de parte dos alunos. Nesse caso, sugerimos a adoção do cadastro do Auxílio Emergencial - benefício financeiro destinado a famílias do Cadastro Único para programas sociais, trabalhadores informais, microempreendedores individuais, autônomos e desempregados -, por ser uma base de dados já existente e que alcança mais famílias em situação de vulnerabilidade social.

\subsubsection{Auxílio emergencial aos municípios com Índice de Desenvolvimento Humano (IDH) baixo e muito baixo}

Considerando que existem EEx que não têm recursos para suplementar o valor repassado pelo governo federal e que, em geral, isso ocorre nos locais em que a alimentação escolar cumpre um papel mais importante na garantia do DHAA, sugerimos que a transferência do governo federal para municípios com IDH baixo (entre 0,500 e 0,599) e muito baixo (até 0,499) seja equivalente ao valor per capita transferido para comunidades tradicionais (indígenas, quilombolas) - entre $\mathrm{R} \$ 0,64$ e $\mathrm{R} \$ 1,07$, a depender da modalidade de ensino e período escolar. No Brasil, há 1399 municípios com IDH abaixo de 0,600 (32 classificados como muito baixo), majoritariamente concentrados em áreas rurais e na região Nordeste. Segundo o Censo Escolar de 2019, esses municípios atendiam 6.272.164 $(17,1 \%)$ escolares da educação básica do total (PNUD, 2013).

Desse modo, com um aumento de apenas 3\% no orçamento total do programa (Tabela 1), esses municípios terão mais recursos para que a distribuição de kits ou refeições seja mantida para todos os estudantes.

O auxílio emergencial para municípios com IDH baixo e muito baixo tende a beneficiar apenas municípios menores, não atingindo os bolsões de pobreza em áreas metropolitanas. Em que pese a necessidade de aumentar o repasse a todos os municípios com alunos em situação de IA - como propomos nas estratégias a serem implementadas após o retorno às aulas -, é preciso destacar que municípios maiores e com maior IDH contam com maior arrecadação de impostos e, consequentemente, maior disponibilidade orçamentária para complementar os recursos advindos do PNAE (Castro, Guimarães, Paula, Souza, \& Fully, 2018).

ReVISta de Administração Pública | Rio de Janeiro 54(4):1134-1145, jul. - ago. 2020 
TABELA 1

\title{
DADOS SOBRE A ALIMENTAÇÃO ESCOLAR E ESTIMATIVAS SOBRE TOTAL DE RECURSOS NECESSÁRIOS PARA AMPLIAÇÃO DO PNAE
}

\author{
Dados sobre alimentação escolar e escolares \\ Números \\ Total de recurso utilizado pelo PNAE em $2019(\mathrm{R} \$)^{*}$ \\ $3.950 .473 .553,79$ \\ Total de escolares da educação básica pública** \\ 36.611 .223 \\ Total de estudantes da educação básica em municípios classificados com IDH Baixo e Muito Baixo ${ }^{\star \star \star ~}$ \\ Valor do médio do repasse financeiro do FNDE para o PNAE, por dia e estudante da educação básica (R\$) \\ 6.272 .164 \\ 0,54 \\ Impacto financeiro no aumento do valor diário por estudante do PNAE, destinado aos municípios de \\ $125.443 .280,00$ \\ baixo e muito baixo IDH $(\mathrm{R} \$)^{t}$ \\ Impacto financeiro no aumento do valor diário por estudante do PNAE e ampliação do benefício para \\ 0 ano inteiro, destinado aos municípios de baixo e muito baixo IDH $(\mathrm{R} \$)^{H}$ \\ PNAE - Programa Nacional de Alimentação Escolar / FNDE - Fundo Nacional para o Desenvolvimento da Educação do Ministério \\ da Educação. \\ * Dados extraídos do sistema do sistema de informações de liberação de recursos do FNDE (Recuperado de https://www.fnde.gov.br/ \\ sigefweb/index.php/liberacoes). \\ ** Dados extraídos do Censo Escolar da Educação em 2019 (Recuperado de http://portal.inep.gov.br/censo-escolar). \\ *** Dados obtidos Índice de Desenvolvimento Humano Municipal Brasileiro (PNUD, 2013). \\ $¥$ Aumento do repasse do valor por estudante por dia para $\mathrm{R} \$ 0,64$, para os dias letivos. \\ \#ł Aumento do repasse do valor por estudante por dia para $\mathrm{R} \$ 0,64$, para o ano inteiro. \\ Fonte: Elaborada pelos autores.
}

\subsubsection{Manutenção e incentivo à aquisição de alimentos da agricultura familiar}

Estudos apontam que a inserção da AF no PNAE promoveu um aumento na renda familiar, com a garantia de acesso ao mercado e o escoamento da produção, o que tem interferido positivamente na SAN dos agricultores (Assis, Priore, \& Franceschin, 2017). Além disso, os produtos da AF contribuem para a qualidade da alimentação escolar, por serem alimentos in natura e minimamente processados (Teo, 2018). O próprio FNDE se preocupa com esse público neste momento, e a Cartilha com Orientações do PNAE durante a pandemia aponta novas formas de execução, apresentando como estratégia a continuidade da compra da agricultura familiar e a elaboração da chamada pública eletrônica. Há ainda a orientação da manutenção dos contratos firmados com os agricultores familiares, respeitando o calendário de entrega já estipulado, de modo que o agricultor não seja onerado (Mapa \& MEC, 2020).

Cabe ainda destacar que o incentivo à aquisição de alimentos da AF com recursos do PNAE depende também da coordenação das ações governamentais para superar as dificuldades enfrentadas pelo PAA. Sambuichi, Galindo, Oliveira, e Moura (2014) apontam algumas medidas que podem ser tomadas para fortalecer esse mercado de compras públicas da AF: melhoria da estrutura logística de apoio a essas compras (transporte, beneficiamento e armazenamento), promoção de assistência técnica continuada que forneça apoio ao planejamento da unidade produtiva familiar de forma integrada e a garantia de crédito de maneira adequada às necessidades do pequeno produtor. Essas ações podem e devem ir além da etapa da pandemia, contribuindo para a ampliação das compras da AF no Programa. 


\subsection{Estratégias a serem implementadas após o retorno às aulas}

\subsubsection{Levantamento da IA entre os alunos}

Neste momento de crise, fica ainda mais evidente a importância dos dados para guiar as ações do poder público. Assim, sugerimos que a versão curta da Ebia seja aplicada no ato da matrícula/ rematrícula dos alunos. Essa versão conta com 5 perguntas autoaplicáveis, de fácil resposta, permitindo um diagnóstico das famílias dos escolares em insegurança alimentar e nutricional (Santos et al., 2014). Sugere-se que esse dado seja declarado pelas escolas todos os anos, juntamente com o censo escolar, medida que seria simples e sem impacto no custo, permitindo um diagnóstico da proporção de estudantes em famílias com IA por escola. Ressalta-se que as escalas de medida direta da insegurança alimentar, como a Ebia, fornecem informações estratégicas para a gestão de políticas e programas sociais em nível nacional e municipal, pois permitem tanto identificar e quantificar os grupos sociais em risco de insegurança alimentar quanto seus determinantes e consequências (Traldi \& Almeida, 2012).

\subsubsection{Ampliação do valor per capita repassado}

Os dados obtidos com a aplicação da Ebia permitirão elaborar um diagnóstico para avaliação e aperfeiçoamento da política, bem como ajudar na tomada de decisões em outros momentos de crise. Sugerimos a ampliação do valor per capita diário repassado a cada aluno em situação de IA, ampliando a equidade dentro do PNAE. Recomendamos a utilização do mesmo valor repassado atualmente a estudantes indígenas e quilombolas - $\mathrm{R} \$ 0,64$ para estudantes de meio período e $\mathrm{R} \$ 1,07$ para estudantes de período integral.

Com os dados sobre a IA em seu território, as gestões estaduais e municipais terão condições de direcionar esses valores complementares às escolas com maiores índices de alunos nessa situação.

\subsubsection{Atendimento dos escolares durante as férias e recesso escolar}

Os recursos do PNAE não preveem o fornecimento da alimentação escolar nos períodos de recesso e férias escolares. $\mathrm{O}$ anúncio da suspensão das aulas por conta da pandemia de coronavírus explicitou que, para muitas famílias, a interrupção do fornecimento da alimentação escolar implica o agravamento da situação de insegurança alimentar.

O aumento do desemprego e da pobreza em consequência da pandemia ampliará o número de domicílios em IA. Assim, propomos a manutenção do atendimento aos escolares durante as férias e o recesso escolar após o fim da pandemia. Caso seja realizado o levantamento da IA entre os alunos, poderiam ser elegíveis famílias a receber a ampliação. Sem a realização do levantamento, propomos atender todas as escolas em municípios com IDH baixo e muito baixo. Tal ampliação de dias levaria ao orçamento do PNAE um gasto adicional aproximado de $\mathrm{R} \$ 788$ milhões, em torno de $20 \%$ do orçamento total do programa em 2019 (Tabela 1).

A forma de fornecimento da alimentação escolar durante as férias/ recesso escolar, ficará a cargo da gestão da EEx, seja com os kits de alimentos, seja com a manutenção da refeição diária na escola. Essa ação poderá ressignificar o papel da escola e sua relação com a comunidade. 


\subsubsection{Manutenção da equidade do PNAE}

Propomos que o Auxílio Emergencial aos municípios com IDH baixo e muito baixo seja mantido após a pandemia, garantindo que eles continuem recebendo valores per capita equivalentes aos transferidos a comunidades tradicionais e, consequentemente, ampliando a equidade do programa. Tal ação deverá ser mantida enquanto não estiverem disponíveis os dados de IA nos municípios e nas escolas.

\section{CONCLUSÃo}

O PNAE pode ser considerado uma das políticas públicas mais exitosas do país. A um custo de pouco mais de cem reais por ano por estudante, beneficia diretamente, com uma ou mais refeições diárias, mais de 40 milhões de escolares. Ainda assim, o programa enfrenta muitos desafios para cumprir seus objetivos e diretrizes, entre elas a garantia da segurança alimentar e nutricional dos alunos.

Desde o início da pandemia, muitos estados e municípios têm executado o PNAE de uma forma que remete aos primórdios do programa, quando apenas determinados públicos eram atendidos, o que reforçava seu caráter assistencialista.

Nossa proposta, ao contrário, tem como fundamento a defesa do direito à alimentação escolar para toda a educação básica, conquistado há pouco mais de dez anos. Algumas medidas são necessárias para garantir a equidade do programa durante e após a pandemia, dado que as desigualdades econômicas e sociais entre (e internamente aos) estados e municípios são um obstáculo para o cumprimento das diretrizes do PNAE.

A proposta que apresentamos não tem como objetivo superar a totalidade dos desafios a fim de garantir a segurança alimentar e nutricional das famílias com crianças em idade escolar. Ela foi elaborada com base no momento que vivemos, marcado por uma pandemia e pela calamidade pública e crise econômica dela decorrente. O sentido de nossa exposição foi demonstrar como instrumentos como kits de alimentos e/ou refeições, auxílio emergencial aos municípios com IDH baixo e muito baixo, incentivo à aquisição de alimentos da $\mathrm{AF}$, levantamento da IA entre os alunos e ampliação do valor repassado aos estudantes nessa situação e o atendimento durante as férias e recesso escolar permitem ampliar a equidade dentro do programa de maneira ágil e apropriada.

A necessidade de isolamento social também evidenciou que a ampliação do acesso à alimentação para além dos dias letivos, apesar de elevar os gastos do PNAE, tem alto potencial de efetividade na promoção da segurança alimentar e nutricional entre crianças e adolescentes em todo o país.

Desse modo, entendemos que os desafios impostos pela pandemia (e suas consequências econômicas e sociais) para a execução do PNAE devem ser encarados, ao mesmo tempo, como um chamado para um debate mais amplo e profundo sobre o sentido e como o significado da alimentação escolar num país com altos índices de insegurança alimentar. 


\section{REFERÊNCIAS}

Assis, S. C. R, Priore, S. E., \& Franceschin, S. C. C. (2017). Impacto do Programa de Aquisição de Alimentos na segurança alimentar e nutricional dos agricultores. Ciências \& Saúde Coletiva, 22(2), 617-626.

Bickel, G., Nord, M., Price, C., Hamilton, W., \& Cook, J. (2000). Guide to measuring household food security, Revised 2000. Alexandria, VA: USDA/Food and Nutrition Service.

Castro, A. V., Guimarães, A. A. B, Paula, J. V, Souza, S, M., \& Fully, R. M. P. (2018). A influência da arrecadação própria municipal sobre o IDH dos municípios mineiros. Revista Científica Doctum Multidisciplinar, 1(1), 1-25.

Fundo Nacional de Desenvolvimento da Educação. (2020). Alimentação escolar: consultas. Dados da Agricultura Familiar. Recuperado de http://www. fnde.gov.br/index.php/programas/pnae/pnaeconsultas/pnae-dados-da-agricultura-familiar

Hoffmann, R. (2012). Determinantes do consumo da merenda escolar no Brasil: análise dos dados da PNAD de 2004 e 2006. Segurança Alimentar e Nutricional, 19(1), 33-45.

Instituto Brasileiro de Geografia e Estatística. (2014). Pesquisa Nacional por Amostra de Domicílios: Segurança Alimentar (2013). Rio de Janeiro, RJ: IBGE.

Instituto Brasileiro de Geografia e Estatística. (2019). Síntese dos indicadores sociais: uma análise das condições de vida da população brasileira. Rio de Janeiro, RJ: IBGE.

Lei $n^{\circ}$ 8.913, de 12 de julho de 1994. (1994). Dispõe sobre a municipalização da merenda escolar. Brasília, DF. Recuperado de www.planalto.gov.br/ccivil_03/ Leis/L8913.htm

Lei $n^{\circ}$ 11.346, de 15 de setembro de 2006. (2006). Cria o Sistema Nacional de Segurança Alimentar e Nutricional (Sisan) com vistas a assegurar o direito humano à alimentação adequada e dá outras providências. Brasília, DF. Recuperado de http://www.planalto.gov.br/ccivil_03/_Ato20042006/2006/Lei/L11346.htm

Lei $n^{\circ} 11.947$, de 16 de junho de 2009. (2009). Dispõe sobre o atendimento da alimentação escolar e do
Programa Dinheiro Direto na Escola aos alunos da educação básica. Brasília, DF. Recuperado de http://www.planalto.gov.br/ccivil_03/_Ato20072010/2009/Lei/L11947.htm

Lei $n$ 13.987, de 7 de abril de 2020. (2020). Altera a Lei $n^{\circ} 11.947$ de 2009 para autorizar, em caráter excepcional, durante o período de suspensão das aulas em razão de situação de emergência ou calamidade pública, a distribuição de gêneros alimentícios adquiridos com recursos do PNAE aos pais ou responsáveis dos estudantes das escolas públicas da educação básica. Brasília, DF. Recuperado de http://www.planalto.gov.br/ ccivil_03/_ato2019-2022/2020/lei/113987.htm

Locatelli, N. T., Canella, D. S., \& Bandoni, D. H. (2017). Fatores associados ao consumo da alimentação escolar por adolescentes no Brasil: resultados da PeNSE 2012. Caderno de Saúde Pública, 33(4), e00183615.

Medida Provisória $n^{\circ}$ 1.979-19, de 2 de junho de 2000. (2000). Dispõe sobre o repasse de recursos financeiros do Programa Nacional de Alimentação Escolar, institui o Programa Dinheiro Direto na Escola e dá outras providências. Brasília, DF.

Ministério da Agricultura, Pecuária e Abastecimento e Ministério da Educação. (2020). Orientações para a execução do PNAE Durante a situação de emergência decorrente da Pandemia do Coronavírus (covid-19). Brasília, DF: Autor.

Peixinho, A. M. L. (2013). A trajetória do Programa Nacional de Alimentação Escolar no período de 2003-2010: relato do gestor nacional. Ciência \& Saúde Coletiva, 18(4), 909-916.

Programa das Nações Unidas para o Desenvolvimento. (2013). Indice de Desenvolvimento Humano Municipal Brasileiro. Brasília, DF: PNUD, IPEA, FJP.

Radimer, K. L., Olson, C. M., Greene, J. C., Campbell, G. C., Habicht, J. P. (1992). Understanding hunger and developing indicators to assess it in women and children. Journal of Nutrition Education, 24(1), 36S-44S.

Resolução CD/FNDE/MEC $n^{\circ} 1$, de 8 de fevereiro de 2017. (2017). Altera o valor per capita para oferta da alimentação escolar do Programa de Alimentação Escolar (PNAE). Brasília, DF. 
Resolução CD/FNDE/MEC no 2, de 9 de abril de 2020. (2020). Dispõe sobre a execução do Programa Nacional de Alimentação Escolar (PNAE) durante o período de estado de calamidade pública, reconhecido pelo Decreto Legislativo ${ }^{\circ} 6$, de 20 de março de 2020, e da emergência de saúde pública de importância internacional decorrente do novo coronavírus (Covid-19). Brasília, DF.

Sambuichi, R. H. R., Galindo, E. P., Oliveira, M. A. C., \& Moura, A. M. M. (2014). Compras públicas sustentáveis e agricultura familiar: a experiência do Programa de Aquisição de Alimentos (PAA) e do Programa Nacional de Alimentação Escolar (PNAE). In R. H. R. Sambuichi, E. P. Galindo, M. A. C. Oliveira, \& A. M. M. Moura. (Orgs.), Politicas agroambientais e sustentabilidade: desafios, oportunidades e lições aprendidas. Brasília, DF: Instituto de Pesquisa Econômica Aplicada.

Santos, L. P., Lindemann, I. L., Mottal, J. V. S, Mintem, G., Benderl, E., \& Gigante, D. P. (2014). Proposta de versão curta da Escala Brasileira de Insegurança Alimentar. Revista de Saúde Pública, 48(5),783-789.
Segal-Corrêa, A. M., \& Marin-Leon, L. (2009). A segurança alimentar no Brasil: proposição e usos da Escala Brasileira de Medida da Insegurança Alimentar (Ebia) de 2003 a 2009. Segurança Alimentar e Nutricional, 16(2), 1-19.

Teo, C. R. P. A. (2018). The partnership between the Brazilian School Feeding Program and family farming: a way for reducing ultra-processed foods in school meals. Public Health Nutricion, 21(1), 230-237.

Traldi, D. R. C., \& Almeida, L. L. M. C. (2012). Políticas públicas de transferência de renda e a questão da segurança alimentar dos beneficiários: efetividades e entraves do Programa Bolsa Família. Política \& Sociedades, 11(21),137-171.

Vasconcelos, F. (2005). Combate à fome no Brasil: uma análise histórica de Vargas a Lula. Revista Nutrição, 4(18), 439-457.

Wehler, C. A., Scoot, R. I., \& Anderson, J. J. (1992). The community childhood hunger identification project: a model of domestic hunger-demonstration project in Seattle, Washington. Journal of nutrition education, 24(1), 29S-35S.

\section{Ana Laura Benevenuto de Amorim}

https://orcid.org/0000-0003-0745-8201

Nutricionista; Doutoranda pelo Programa Interdisciplinar em Ciências da Saúde da Universidade Federal de São Paulo (UNIFESP), Campus Baixada Santista. E-mail: analauraba@hotmail.com

\section{José Raimundo Sousa Ribeiro Junior}

https://orcid.org/0000-0002-6502-616X

Professor Visitante da Universidade Federal de São Paulo (UNIFESP) no Centro de Práticas e Pesquisa em Nutrição e Alimentação Coletiva (CPPNAC). E-mail: sousaribeirojunior@gmail.com

\section{Daniel Henrique Bandoni}

https://orcid.org/0000-0003-1638-1437

Professor Associado do Curso de Nutrição da Universidade Federal de São Paulo (UNIFESP) pelo Departamento de Saúde, Clínica e Instituições no Instituto de Saúde e Sociedade, Campus Baixada Santista.

E-mail: danielbandoni@gmail.com 